Prospect. Univ. (2010)

\title{
DENSIDAD DE SIEMBRA EN EL POTENCIAL DE RENDIMIENTO DE CEBADA DÍSTICA (Hordeum distichon L.) CENTENARIO CON SIEMBRA TARDÍA EN CONDICIONES DE SECANO ${ }^{29}$
}

Aquino Zacarias, Vidal César1, Yupanqui Rivas, Fredy Fernando²

Facultad de Agronomía de la Universidad Nacional del Centro del Perú

\begin{abstract}
RESUMEN
La cebada dística Centenario, se viene conduciendo por dos campañas agrícolas para alcanzar adaptabilidad plena con alta capacidad de rendimiento, cuidando de no variar las características de grano y sin perder su comportamiento de evasión a factores que limitan el incremento de la productividad. El estudio comprendió la segunda fase comparando 4 densidades poblacionales en condiciones de siembra tardía y en secano. El ensayo fue instalado en BCA con 3 repeticiones en la EEAM, UNCP, con el objetivo; de determinar el efecto de la densidad poblacional en el potencial de rendimiento y compensación de sus componentes de rendimiento con siembra tardía en secano en el valle del Mantaro. Se ejecutó la siembra en hileras. En respuesta de la población de plantas para adaptación, utilizando para la siembra 270 semillas por hilera (90 kg/ha) se alcanzó mayor precocidad, necesitando 63,33 días al 50\% de floración, logrando mayor longitud de espiga con $8,667 \mathrm{~cm}$. Considerando evitar el tumbado, la menor altura de plantas se logró utilizando 450 semillas por hilera (150 kg/ha) con 101,9 cm. De los componentes de rendimiento, la densidad 270 semillas por hilera (90 kg/ha) alcanzó mayor establecimiento con 514,3 espigas por $\mathrm{m}^{2}$, obtuvo además 23,53 granos por espiga. Utilizando 360 semillas por hilera (120 kg/ha), se logró mejor llenado de granos con $71,47 \mathrm{~g}$ por mil granos $(0,07147 \mathrm{~g}$ por grano). El mayor rendimiento potencial en campo, se obtuvo utilizando 270 semillas por hilera $(90 \mathrm{~kg} / \mathrm{ha}$ ) con $4338,33 \mathrm{~kg} / \mathrm{ha}$ y en los componentes de rendimiento, se obtuvo sembrando 360 semillas por hilera (120 kg/ha), con 8083,41 kg/ha.
\end{abstract}

Palabras clave: (población, rendimiento, precocidad, adaptación)

\section{PLANTING DENSITY ON YIELD POTENTIAL OF BARLEY Statistics ( hordeum distichon I.) CENTENNIAL WITH LATE SEEDING UPLAND CONDITIONS}

\begin{abstract}
Centenariol statistical barley, has been driving for two seasons to reach full fitness with high performance capability, being careful not to change the characteristics of grain and without losing their avoidance behavior to factors limiting productivity growth. The study involved the second stage comparing 4 population densities in late planting conditions and dry. The experiment was installed in BCA with 3 replications in EEAM, UNCP, with the aim: to determine the effect of population density on yield potential and compensation components of performance in rainfed late planting in the Mantaro Valley. Ran row planting. In response to plant population adjustment, using planting 270 seeds per row (90 kg / ha) was reached early flowering, necessitating 63.33 days to $50 \%$ flowering, achieving greater spike length with $8.667 \mathrm{~cm}$. Whereas avoid lying, lower plant height was achieved using 450 seeds per row (150 kg / ha) to $101.9 \mathrm{~cm}$. Performance components, the density 270 seeds per row (90 kg / ha) achieved greater facility with 514.3 spikes per m2, also obtained 23.53 grains per spike. Using 360 seeds per row (120 kg / ha) was achieved better grain filling with 71.47 grams per thousand grains $(0.07147$ grams per grain). Higher yield potential in the field, was obtained using 270 seeds per row (90 kg / ha) to $4338.33 \mathrm{~kg} / \mathrm{ha}$ and yield components were obtained planting 360 seeds per row $(120 \mathrm{~kg} / \mathrm{ha}) 8083,41 \mathrm{~kg} / \mathrm{ha}$.
\end{abstract}

Keywords: population, yield, earliness, adaptation

${ }_{1}^{1}$ Docente principal, Facultad de Agronomía, UNCP. E-Mail: cesaraquinozac@hotmail.com

2 Docente principal, Facultad de Agronomía, UNCP. E-mail: frivasyupanqui@yahoo.es

29 Trabajo de investigación fue recibido el 07/05/2010 retornado para su revisión el 15/06/2010 y aprobado para su publicación 16/11/2010. 


\section{INTRODUCCIÓN}

Son preocupantes los efectos del calentamiento global en el valle del Mantaro, pues el distanciamiento del establecimiento de lluvias con presencia frecuente de sequías, hace que la época de siembra propicia, se vea alterada. En cebada, las fechas propicias fueron de octubre a diciembre, que obligados por el clima cambiante, se está sembrando muy tarde con el riesgo de no coincidir con un clima benigno al momento del llenado de grano. Además se observa deficiencias en el uso conveniente de semillas por unidad de superficie, obligando a contar con poblaciones muy bajas o muy altas conllevando a manejar deficientemente la evapotranspiración, siendo afectados los componentes de rendimiento. La variedad CENTENARIO, ofrece una alternativa de solución a las pocas variedades que cuenta el medio y con tolerancia a la poca disponibilidad de agua. Se planteó el presente trabajo con el objetivo de determinar el efecto de la densidad poblacional en el potencial de rendimiento y compensación de sus componentes de rendimiento en la cebada dística Centenario introducida y adaptada con siembra tardía en condiciones de secano en el valle del Mantaro.

\section{MATERIALES Y MÉTODOS}

\section{Lugar experimental}

Lote 5 de la EEA "El Mantaro", campaña agrícola 20072008, distrito, El Mantaro, provincia, Jauja, departamento, Junín. Carretera Central, km 34, margen izquierda; latitud sur, 12 $03^{\prime} 19^{\prime \prime}$; longitud oeste, 75¹6'33"; altitud, 3316 msnm.

\section{Fecha de inicio y culminación del experimento}

Siembra en enero 4 de 2008, culminando con la cosecha el 26 en junio de 2008.

\section{Técnicas del estudio}

El experimento se instaló en hileras de $4 m$ (2 hileras/parcela), comparando densidades poblacionales en base al número de granos, la selección entre densidades poblacionales, se realizó por sus características agronómicas con siembra bajo condiciones de época tardía:

\section{Días al $\mathbf{5 0 \%}$ de floración (DF)}

Indica la precocidad. Se contabilizó los días transcurridos desde la siembra hasta alcanzar el $50 \%$ de floración (antesis: estadío 6 de la escala).

\section{Altura de plantas (AP)}

Evaluada desde el cuello de la planta (base) hasta el final del pedúnculo floral. Se evaluó al final de la maduración del grano (estadio: 87, masa dura en el grano).

\section{Longitud de espiga (LE)}

Evaluada desde la base de la espiga hasta la espiguilla superior de la espiga sin considerar las aristas, al mismo estadío de altura de plantas.

\section{Espigas por $m^{2}\left(\mathrm{Em}^{2}\right)$}

Primer componente, se midió un metro lineal, en toda la longitud y se contabilizó las espigas. Cada muestra representó el $0,3 m^{2}$ con este dato se calculó espigas por $m^{2}$.

\section{Granos por espiga (GE)}

Segundo componente. Se contabilizaron los granos por separado (previa trilla de la espiga individual) y se sumaron los datos para determinar el promedio.

\section{Peso de mil granos (PMG)}

Tercer componente. Se contó 250 granos por muestra (dos muestras), se pesó en forma individual, se sumó y se multiplicó por 2. El resultado indica el llenado del grano.

\section{Rendimiento (RP)}

Característica obtenida con resultados a partir de la cosecha de la parcela $\left(2,4 m^{2}\right)$, se determinó el rendimiento de grano por parcela individual.

\section{Factores en estudio}

Densidad poblacional (dosis de siembra): $T_{1}=270$ semillas (90 kg/ha). $T_{2}=360$ semillas (120 kg/ha). $T_{3}=450$ semillas (150 kg/ha). $T_{4}=540$ semillas (180 kg/ha).

\section{Diseño de investigación}

Modelo de Regresión. El modelo regresional ajustado es el lineal

$$
\begin{gathered}
Y_{i}=\mu+\beta\left(X_{i}-X\right)+\xi_{j} \\
\text { Ecuación: } Y=\beta_{0}+\beta_{1} X
\end{gathered}
$$

\section{Diseño bloques completos al azar}

Modelo aditivo lineal (Cochran y Cox, 1990): $Y_{i j}=\mu+\zeta_{i}+$ $\beta_{\mathrm{j}}+\varepsilon_{\mathrm{ij}}$

\section{RESULTADOS}

Respuesta de la población de plantas con siembra tardía sobre características de adaptación

Cuadro 1. Análisis de variancia para características de adaptación. 


\begin{tabular}{|c|c|c|c|c|}
\hline \multirow[b]{2}{*}{ FV } & \multicolumn{4}{|c|}{ Características de adaptación (CM) } \\
\hline & GL & $\begin{array}{l}\text { Dias } 50 \% \\
\text { floración }\end{array}$ & $\begin{array}{c}\text { Altura de } \\
\text { planta }(\mathrm{cm})\end{array}$ & $\begin{array}{l}\text { Longitud de } \\
\text { espiga }(\mathrm{cm})\end{array}$ \\
\hline Bloques & 2 & $19,750 \mathrm{~ns}$ & $26,670 \mathrm{~ns}$ & 0,033 ns \\
\hline Tratamientos & 3 & 73,000 ** & $40,919 \mathrm{~ns}$ & $0,168 \mathrm{~ns}$ \\
\hline \multirow[t]{3}{*}{ Error } & 6 & 6,750 & 61,972 & 0,076 \\
\hline & Promedio & 69,5 & 105,850 & 8,351 \\
\hline & CV (\%) & 3,74 & 7,44 & 3,29 \\
\hline
\end{tabular}

CM: cuadrado medio del error. **: altamente significativo. ns: no significativo

Cuadro 2. Prueba de significación de las características de adaptación.

\begin{tabular}{cccccccccc}
\hline & \multicolumn{3}{c}{$\begin{array}{c}\text { Días al } 50 \% \text { de } \\
\text { floración }\end{array}$} & \multicolumn{3}{c}{$\begin{array}{c}\text { Alturas de plantas } \\
(\mathrm{cm})\end{array}$} & \multicolumn{3}{c}{$\begin{array}{c}\text { Longitud de espiga } \\
(\mathrm{cm})\end{array}$} \\
\cline { 2 - 9 } $\mathrm{M}$ & $\mathrm{T}$ & $\mathrm{x}$ & $\mathrm{si}$ & $\mathrm{T}$ & $\mathrm{x}$ & $\mathrm{si}$ & $\mathrm{T}$ & $\mathrm{x}$ & $\mathrm{sig}$ \\
& & & $\mathrm{g}$ & & & $\mathrm{g}$ & & & \\
1 & $\mathrm{~T}_{4}$ & 75,33 & $\mathrm{a}$ & $\mathrm{T}_{1}$ & 109,8 & $\mathrm{a}$ & $\mathrm{T}_{1}$ & 8,667 & $\mathrm{a}$ \\
2 & $\mathrm{~T}_{3}$ & 70,33 & $\mathrm{ab}$ & $\mathrm{T}_{2}$ & 108,1 & $\mathrm{a}$ & $\mathrm{T}_{3}$ & 8,333 & $\mathrm{a}$ \\
3 & $\mathrm{~T}_{2}$ & 69,00 & $\mathrm{ab}$ & $\mathrm{T}_{4}$ & 103,6 & $\mathrm{a}$ & $\mathrm{T}_{2}$ & 8,310 & $\mathrm{a}$ \\
4 & $\mathrm{~T}_{1}$ & 63,33 & $\mathrm{~b}$ & $\mathrm{~T}_{3}$ & 101,9 & $\mathrm{a}$ & $\mathrm{T}_{4}$ & 8,093 & $\mathrm{a}$
\end{tabular}

\begin{tabular}{rrrr} 
Sx & 1,5 & 4,545 & 0,1592 \\
\hline
\end{tabular}

$\mathrm{T}$ : tratamientos. $\mathrm{x}$ : promedio. sig: significación estadística según Tukey $(0,05)$. Datos originales.

Cuadro 3. Regresión lineal de características de adaptación con el rendimiento.

\begin{tabular}{|c|c|c|c|c|}
\hline FV & GL & $\mathrm{DF}(\mathrm{CM})$ & $\mathrm{AP}(\mathrm{CM})$ & LE (CM) \\
\hline Regresión & 1 & $164334,874 \mathrm{~m} n s$ & 270733,924 & 151455,373 \\
\hline Residual & 2 & 65218,135 & * & ns \\
\hline Total & 3 & 8257,048 & $\begin{array}{l}12018,610 \\
98257,048\end{array}$ & 71657,886 \\
\hline
\end{tabular}

98257,048

DF: 50\%, floración. AP: altura, planta. LE: longitud (espiga).

CM: cuadrado medio del error. $F \alpha_{(0,05): 18,51 \text {. } F a(0,01): 98,5}$

\section{Respuesta de la población de plantas con siembra tardía sobre los componentes de rendimiento}

Cuadro 4. Análisis de variancia para los componentes de rendimiento.

\begin{tabular}{|c|c|c|c|}
\hline \multirow{2}{*}{ FV } & \multicolumn{3}{|c|}{ Componentes de rendimiento (CM) } \\
\hline & Espigas $/ \mathrm{m}^{2}$ & $\begin{array}{c}\text { Granos/espi } \\
\text { ga }\end{array}$ & Peso de grano $(\mathrm{g})$ \\
\hline $\begin{array}{l}\text { Bloques } \\
\text { Tratamientos } \\
\text { Error }\end{array}$ & $\begin{array}{l}2885,583 \mathrm{~ns} \\
12632,00 \mathrm{~ns} \\
15572,250\end{array}$ & $\begin{array}{l}0,603 \mathrm{~ns} \\
1,029 \mathrm{~ns} \\
0,786\end{array}$ & $\begin{array}{l}4,02088 \times 10^{-5} \mathrm{~ns} \\
1,54800 \times 10^{-5} \mathrm{~ns} \\
1,05170 \times 10^{-5}\end{array}$ \\
\hline $\begin{array}{l}\text { Promedio } \\
\text { CV (\%) }\end{array}$ & $\begin{array}{c}451,667 \\
27,63\end{array}$ & $\begin{array}{c}23,033 \\
3,85\end{array}$ & $\begin{array}{l}0,069 \\
4,70\end{array}$ \\
\hline
\end{tabular}

CM: cuadrado medio del error. ns: no significativo
Cuadro 5. Prueba de significación de los promedios de los componentes de rendimiento.

\begin{tabular}{cccccccccc}
\hline \multirow{2}{*}{ OM } & \multicolumn{3}{c}{ Espigas $/ m^{2}$} & \multicolumn{4}{c}{ Granos/espiga } & \multicolumn{3}{c}{ Peso de grano } \\
\cline { 2 - 10 } & $\mathrm{T}$ & $\mathrm{x}$ & sig & $\mathrm{T}$ & $\mathrm{x}$ & sig & $\mathrm{T}$ & $\mathrm{x}$ & sig \\
\hline 1 & $\mathrm{~T}_{1}$ & 514,3 & $\mathrm{a}$ & $\mathrm{T}_{1}$ & 23,53 & $\mathrm{a}$ & $\mathrm{T}_{2}$ & 0,07147 & $\mathrm{a}$ \\
2 & $\mathrm{~T}_{2}$ & 483,7 & $\mathrm{a}$ & $\mathrm{T}_{2}$ & 23,50 & $\mathrm{a}$ & $\mathrm{T}_{4}$ & 0,07073 & $\mathrm{a}$ \\
3 & $\mathrm{~T}_{4}$ & 444,3 & $\mathrm{a}$ & $\mathrm{T}_{3}$ & 22,77 & $\mathrm{a}$ & $\mathrm{T}_{3}$ & 0,06780 & $\mathrm{a}$ \\
4 & $\mathrm{~T}_{3}$ & 364,3 & $\mathrm{a}$ & $\mathrm{T}_{4}$ & 22,33 & $\mathrm{a}$ & $\mathrm{T}_{1}$ & 0,06673 & $\mathrm{a}$ \\
$\mathrm{Sx}$ & & 72,05 & & & 0,5119 & & & 0,001826 & \\
\hline
\end{tabular}

$\mathrm{T}$ : tratamientos. $\mathrm{x}$ : promedio. sig: significación estadística según Tukey $(0,05)$. Datos originales.

Cuadro 6. Datos de regresión lineal de los componentes de rendimiento con el rendimiento.

\begin{tabular}{lclcc}
\hline \multicolumn{1}{c}{ FV } & GL & Espigas $/ \mathrm{m}^{2}(\mathrm{CM})$ & $\begin{array}{c}\text { Granos/espiga } \\
(\mathrm{CM})\end{array}$ & $\begin{array}{c}\text { Peso de } \\
\text { grano }(\mathrm{CM})\end{array}$ \\
\hline Regresión & 1 & $265690,638 \mathrm{~ns}$ & $157515,600 \mathrm{~ns}$ & 18149,485 \\
Residual & 2 & 14540,254 & 68627,773 & $\mathrm{~ns}$ \\
Total & 3 & 98257,048 & 98257,048 & 138310,831 \\
& & & & 98257,048 \\
\hline
\end{tabular}

CM: cuadrado medio del error. $\mathrm{Fa}_{(0,05)}: 18,51$. $\mathrm{Fa}_{(0,01)}: 98,5$

\section{Rendimiento potencial}

Cuadro 7. Análisis de variancia para rendimiento potencial.

\begin{tabular}{llll}
\hline \multirow{2}{*}{ FV } & \multicolumn{3}{c}{ Rendimiento potencial de granos (CM) } \\
\cline { 2 - 4 } & GL & \multicolumn{1}{c}{ RPC $\left(\mathrm{g} / 2,4 \mathrm{~m}^{2}\right)$} & \multicolumn{1}{c}{ RPCR $(\mathrm{kg} / \mathrm{ha})$} \\
\hline Bloques & 2 & $8863,360 \mathrm{~ns}$ & $1378919,284 \mathrm{~ns}$ \\
Tratamientos & 3 & $17256,669 \mathrm{~ns}$ & $3644100,280 \mathrm{~ns}$ \\
Error & 6 & 6325,975 & 3466048,070 \\
$\quad$ Promedio & & 943,408 & 7202,523 \\
$\quad$ CV (\%) & \multicolumn{1}{c}{13,54} & 25,85 \\
\hline
\end{tabular}

RPC: rendimiento potencial de campo. CM: cuadrado medio del error. ns: no significativo

Cuadro 8. Prueba de significación de los promedios del rendimiento potencial.

\begin{tabular}{|c|c|c|c|c|c|c|}
\hline \multirow[t]{2}{*}{$\mathrm{OM}$} & \multicolumn{3}{|c|}{$\begin{array}{c}\text { Rendimiento } \\
\text { potencial de campo } \\
\left(\mathrm{g} / 2,4 \mathrm{~m}^{2}\right)\end{array}$} & \multicolumn{3}{|c|}{$\begin{array}{l}\text { Rendimiento potencial de los } \\
\text { componentes de rendimiento } \\
(\mathrm{kg} / \mathrm{ha})\end{array}$} \\
\hline & $\mathrm{T}$ & $x$ & sig & $\mathrm{T}$ & $x$ & sig \\
\hline 1 & $\mathrm{~T}_{1}$ & 1041,2 & $a$ & $\mathrm{~T}_{2}$ & 8083,41 & a \\
\hline 2 & $\mathrm{~T}_{2}$ & 957,0 & $a$ & $\mathrm{~T}_{4}$ & 7969,50 & a \\
\hline 3 & $\mathrm{~T}_{4}$ & 913,3 & $a$ & $\mathrm{~T}_{1}$ & 7057,62 & a \\
\hline 4 & $\mathrm{~T}_{3}$ & 862,2 & $a$ & $\mathrm{~T}_{3}$ & 5699,56 & a \\
\hline Sx & & 73,77 & & & 1075,0 & \\
\hline
\end{tabular}

$\mathrm{T}$ : tratamientos. $\mathrm{x}$ : promedio. sig: significación estadística según Tukey $(0,05)$. Datos originales. 


\section{DISCUSIÓN}

\section{Respuesta de la población de plantas con siembra tardía sobre características de adaptación}

En el cuadro 1, en días al $50 \%$ de floración (precocidad) se observa diferencias estadísticas altamente significativas para densidad poblacional (tratamientos), debido a la respuesta genética al medio donde fue instalado el ensayo, promoviendo a la mayor o menor precocidad. Se necesitó 69,5 días, 3,611 días más con respecto a la campaña agrícola 2006-2007 (65,889 días) sembrado en campaña normal, con coeficiente de variabilidad de $3,74 \%$, calificado excelente la precisión del experimento indicando bastante homogeneidad dentro de cada tratamiento.

En altura de plantas y longitud de espigas, no se encontraron diferencias estadísticas, debido a que éstas características son propias de la respuesta genética antes que agronómico. Se alcanzó 105,85 cm de altura; en longitud de espigas, 8,351 $\mathrm{cm}, 1,537 \mathrm{~cm}$ más pequeña respecto a lo alcanzado en la campaña agrícola anterior con siembra normal $(9,888 \mathrm{~cm})$, debido a la siembra tardía.

El coeficiente de variabilidad fue de $7,44 \%$ para altura de plantas y $3,29 \%$ en longitud de espigas, calificado excelente la precisión del experimento, mostrando ser muy homogéneos dentro de cada tratamiento.

Del cuadro 2, de la prueba de significación según Tukey, para precocidad, se observa que la densidad de siembra 270 semillas ( $\left.T_{1}, 90 \mathrm{~kg} / \mathrm{ha}\right)$ muestra diferencia estadística con las demás densidades. En el cultivar Centenario, para obtener mayor precocidad se debe utilizar menores densidades de siembra, con esta densidad se necesitó 63,33 días para alcanzar el $50 \%$ de floración en condiciones de siembra tardía. Este resultado es debido al uso de densidad baja, promoviendo evapotranspiración mayor, obligando al cultivo acelerar el crecimiento y desarrollo de sus estados fenológicos.

Respecto a altura de plantas como a longitud de espigas, se observa que no existen diferencias estadísticas entre ellas, considerando que menor densidad promueve mayor altura de planta y longitud de espiga, en ambas características $90 \mathrm{~kg} / \mathrm{ha}$ ( $\mathrm{T}_{1}$ : 270 semillas por hilera) motivó alcanzar mayor tamaño con 109,8 cm de altura de planta y $8,667 \mathrm{~cm}$ en longitud de espigas en promedio. Resultado debido al efecto de compensación por parte de la planta cebada dística Centenario, que en condiciones de siembra tardía para evitar el tumbado, necesitó utilizar 450 semillas por hilera $\left(T_{3}: 150\right.$ $\mathrm{kg} / \mathrm{ha}$ ) para alcanzar $101,9 \mathrm{~cm}$. Sin embargo a menores densidades promueve mayor precocidad con mayores tamaños, siendo lo contrario utilizando mayores densidades en condiciones de siembra tardía y en secano.

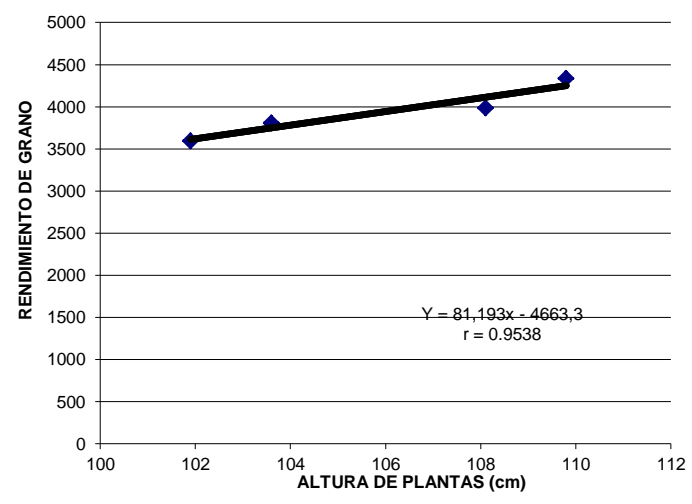

Grafico 1. Regresión lineal de altura de plantas con el rendimiento de grano proveniente de campo.

Del cuadro 3, para días al $50 \%$ de floración y longitud de espigas, no existen diferencias estadísticas significativas para la regresión, no estando correlacionadas con el aumento o disminución del rendimiento. Existe diferencia estadística significativa en altura de plantas, estando correlacionada con el aumento del rendimiento, alcanzando un coeficiente de correlación (r) de 0,9538 (gráfico 1). La ecuación de regresión es, $\hat{Y}=81,193(x)-4663,3$, por el incremento de un centímetro en altura de planta, aumenta el rendimiento de granos en $80,935 \mathrm{~kg} / \mathrm{ha}$. Respuesta que se aprecia en el coeficiente de determinación $\left(R^{2}\right)$ con 0,9097 , donde el $90,97 \%$ del aumento del rendimiento $(\hat{Y})$, se debe a la influencia de la altura de planta. Resultado debido al incremento del tamaño (mayor biomasa) en condiciones de siembra tardía y en secano, el cultivo trata de compensar con mayor producción de fotosintatos a favor del llenado de granos para alcanzar los rendimientos esperados.

\section{Respuesta de la población de plantas con siembra tardía sobre los componentes de rendimiento}

Del cuadro 4, en espigas por $\mathrm{m}^{2}$, granos por espiga y peso de mil granos, no existen diferencias estadísticas significativas. Resultados debido al establecimiento homogéneo de plántulas de cebada por encontrarse el suelo con suficiente humedad (siembra en enero) y debido al efecto compensatorio en determinar sus características eminentemente genéticas en mayor o menor número de espigas por planta. Se alcanzó 451,667 espigas por $\mathrm{m}^{2}, 102$ espigas más que lo alcanzado en la campaña inmediato anterior (349,667 espigas por $\left.\mathrm{m}^{2}\right)$, que asegura buen rendimiento. En granos por espiga, debido al efecto compensatorio a las diferentes densidades de población, se alcanzó 23,033 granos por espiga, 1,856 granos menos que lo hallado en la campaña anterior $(24,889$ granos 
por espiga), que influyó negativamente en el rendimiento, debido al proceso de compensación al mayor número de espigas para evitar la competencia de nutrientes, espacio, luz, etc. y en condiciones de siembra tardía y en secano. Para peso de mil granos, la densidad poblacional influyó en el peso específico del grano, corroborando la influencia de las características genéticas que muestra la cebada Centenario para mostrar el efecto compensatorio. Se obtuvo $69 \mathrm{~g}$ por mil granos (0,069 g por grano), 20,132 g más que lo alcanzado en la campaña agrícola pasada (48,868 g por mil granos), que influyó positivamente en el rendimiento potencial. Respecto al coeficiente de variabilidad, para espigas por $\mathrm{m}^{2}$ fue de $27,63 \%$ considerado mala la precisión del experimento con tendencia a ser heterogénea dentro de cada tratamiento. En granos por espiga y peso de mil granos se halló $3,85 \%$ y $4,7 \%$ respectivamente considerado excelente, siendo muy homogéneo dentro de cada tratamiento.

En el cuadro 5, de la prueba de significación según Tukey de los componentes de rendimiento, no se halló diferencias estadísticas entre los niveles poblacionales sometidas con siembra tardía y en condiciones de secano, debido a la homogeneidad dentro de cada población. Debido al establecimiento de plantas del cultivo, promoviendo óptimo número de espigas, y al efecto compensatorio para con el establecimiento de granos por espiga y en el peso (densidad) del grano. Aseveración que está ligado íntimamente con la menor densidad de población. La menor densidad $\mathrm{T}_{1}$ (90 $\mathrm{kg} / \mathrm{ha}$ : 270 semillas por hilera) alcanzó mejor espigas por $\mathrm{m}^{2}$ con 514,3 además de mejor establecimiento de granos por espiga con 23,53 granos, este último debido a la menor competencia de nutrientes, espacio, luz, etc. Por el efecto compensatorio, esta densidad se ubicó en el último orden en peso de mil granos con 66,73 g (0,0667 g por grano), es decir mayores espigas, mayor competencia, y que lo compensa con mejor establecimiento de grano por espiga.

En el cuadro 6, se aprecia la no existencia de diferencias estadísticas significativas para la regresión en los componentes de rendimiento, indicando que no se encuentran correlacionadas con el aumento o disminución del rendimiento de granos.

\section{Rendimiento potencial}

En el cuadro 7 del análisis de variancia, para el rendimiento potencial de granos por parcela $\left(2,4 \mathrm{~m}^{2}\right)$ y producto de los componentes de rendimiento, no se hallaron diferencias estadísticas significativas en las fuentes de variabilidad. Se alcanzó $943,408 \mathrm{~g}$ por 2,4 m² (3930,87 kg/ha), 3052,46 kg menos que lo alcanzado en la campaña inmediato anterior $(6983,33 \mathrm{~kg} / \mathrm{ha})$ y en el rendimiento de los componentes se obtuvo $7202,523 \mathrm{~kg} / \mathrm{ha}, 2938,183 \mathrm{~kg}$ menos que lo hallado en la campaña anterior $(4264,34 \mathrm{~kg} / \mathrm{ha})$, corroborando que fue influida por el componente peso de mil granos a efecto de la densidad poblacional. Comparando con el rendimiento potencial de campo, el rendimiento potencial producto de los componentes de rendimiento, obtuvo mejor rendimiento, con $3271,653 \mathrm{~kg}$ más, donde el componente espigas por $\mathrm{m}^{2} \mathrm{y}$ granos por espiga influyeron en el mayor rendimiento. El coeficiente de variabilidad fue de $13,54 \%$ y $25,85 \%$, calificado muy buena y mala la precisión del experimento, siendo homogénea y con tendencia a ser heterogéneo respectivamente dentro de los tratamientos en respuesta al rendimiento.

En el cuadro 8, de la prueba de significación según Tukey, las densidades poblacionales no muestran significación estadística. Debido a la respuesta homogénea a la siembra tardía en condiciones de secano. Apreciando que, bajas densidades de siembra, promueve mayores rendimientos, indicando que utilizando la cebada dística Centenario y semienana, sin considerar el efecto de los componentes de rendimiento, permite mejor adaptación a la siembra tardía y en secano, obteniendo mejor rendimiento por mejor distribución y aprovechamiento de los nutrientes provenientes de los fertilizantes. El rendimiento producto de la cosecha directa de campo, 270 semillas por hilera ( $\left.T_{1}: 900 \mathrm{~kg} / \mathrm{ha}\right)$, se adaptó mejor con 1041,2 g por 2,4m² (4338,33 kg/ha), densidad baja que permite mejor aprovechamiento del suelo por menor competencia entre ellas lo que se ve reflejada en el rendimiento alcanzado. En el rendimiento potencial producto de los componentes de rendimiento, 360 semillas por hilera ( $T_{2}: 120 \mathrm{~kg} / \mathrm{ha}$ ) alcanzó mejor rendimiento con $8083,41 \mathrm{~kg} / \mathrm{ha}$, rendimiento que fue influenciado principalmente por el número de espigas por unidad de superficie.

\section{CONCLUSIONES}

- Utilizando a la siembra de 270 semillas por hilera (90 $\mathrm{kg} / \mathrm{ha}$ ) se alcanzó mayor precocidad, necesitando 63,33 días para alcanzar el $50 \%$ de floración, logrando además mayor longitud de espiga con $8,667 \mathrm{~cm}$.

- Considerando evitar el tumbado, la menor altura de plantas se logró utilizando 450 semillas por hilera (150 $\mathrm{kg} / \mathrm{ha}$ ) con $101,9 \mathrm{~cm}$.

- $\quad$ La densidad de población 270 semillas por hilera $(90$ $\mathrm{kg} / \mathrm{ha}$ ) alcanzó mayor establecimiento de plantas logrando 514,3 espigas por $\mathrm{m}^{2}$, obtuvo además 23,53 granos por espiga, ubicándose en primer orden.

- Utilizando 360 semillas por hilera (120 kg/ha), se promovió mejor llenado de granos con $71,47 \mathrm{~g}$ por mil granos $(0,07147 \mathrm{~g}$ por grano).

- El mayor rendimiento potencial proveniente de campo, se logró utilizando 270 semillas por hilera (90 kg/ha) con $1041,2 \mathrm{~g} / 2,4 \mathrm{~m}^{2}(4338,33 \mathrm{~kg} / \mathrm{ha})$.

- El mayor rendimiento potencial proveniente de los componentes de rendimiento se obtuvo sembrando 360 semillas por hilera (120 kg/ha), con $8083,41 \mathrm{~kg} / \mathrm{ha}$. 


\section{REFERENCIAS BIBLIOGRÁFICAS}

- Castro, J. 2005. Efecto en el rendimiento de cebada mutante (Hordeum vulgare L.) cV. UNALM-95 por competencia con la maleza "cebadilla" (Avena fatua L.). Tesis: Facultad de Agronomía, UNCP. Huancayo.

- De La Morena, I. 1984. Análisis del crecimiento y de la producción de granos en los cultivos de cebada. Universidad de Granada. Facultad de Ciencias. España.

- Doto, S. 1989. Influencia de la densidad y distribución de plantas en el crecimiento y rendimiento de cultivares de triticale y de trigo. Universidad de Córdoba. España.

- García, L. \& J. Ramos. 1990. La cebada: Fisiología de la producción de grano. Ministerio de Agricultura. Pesca y Alimentación. Servicio de Extensión Agraria. Editorial Mundi Prensa. Madrid.

- $\quad$ http://es.wikipedia.org/wiki/Cebada

- http://html.rincondelvago.com/la-cebada.html.

- http://www.infoagro.com/herbaceos/forrajes/cebada. $\mathrm{htm}$ : El cultivo de cebada, requerimientos edafoclimáticos ( $1^{\text {a }}$ parte). The barley growing.

- http://www.monografias.com/trabajos35/la-cebada/lacebada.shtml: Actividad agrícola: la cebada.

- López, P., S. Orovitg \& H. López. 1987. Ensayos de dosis de siembra en cebada. Instituto Técnico Agronómico Provincial. Albacete. España.

- Molina, J. 1990. Morfología y desarrollo de la planta. Taxonomía vegetal. En: La cebada. Ministerio de Agricultura. Pesca y Alimentación. Servicio de Extensión Agraria. Editorial Mundi Prensa. Madrid.

- Parsons, L. 1991. Respuesta de la planta a la deficiencia de agua. En: Mejoramiento de plantas en ambientes poco favorables. Ed. By: Meryl Christiansen. Noriega Editores 534 pp. México.

- Seniglabiese, C. \& R. García. 1979. Efecto de la densidad de las plantas sobre la productividad de los cereales con relación a la fertilidad del suelo. Producción Vegetal. Tomo II. INTA. Pergamino.

- Argentina.

- Wall, P. 1988. Diseños Experimentales. Centro Internacional de Mejoramiento de Maíz y Trigo (CIMMYT). México.

- Zadoks, C., T. Chang \& C. Konzak. 1974. A decimal code for the growth stages of cereals. Weed Res. 14: pp. 415-421. 